\title{
Correction to: Measuring quality of life in children with spinal muscular atrophy: a systematic literature review
}

\author{
Shalvaree Vaidya ${ }^{1}$ (D) Stefan Boes $^{2}$
}

Published online: 14 September 2018

(c) Springer Nature Switzerland AG 2018

\section{Correction to: Quality of Life Research https://doi.org/10.1007/s11136-018-1945-x}

In the original publication of the article, the co-author name "Stefan Boes" has been mistakenly missed out and the e-mail address of the corresponding author is shalvaree.vaidya@ stud.unilu.ch. The original article has been corrected.

The original article can be found online at https://doi.org/10.1007/ s11136-018-1945-x.

Shalvaree Vaidya

shalvaree.vaidya@stud.unilu.ch

1 Biogen International, University of Lucerne, Steinhofstrasse

15C, 6005 Lucerne, Switzerland

2 University of Lucerne, Lucerne, Switzerland 